\title{
Domain wall heat conductance in ferromagnetic wires
}

\author{
Peng Yan $^{1}$ and Gerrit E.W. Bauer ${ }^{2,1}$ \\ ${ }^{1}$ Kavli Institute of NanoScience, Delft University of Technology, \\ Lorentzweg 1, 2628 CJ Delft, The Netherlands and \\ ${ }^{2}$ Institute for Materials Research, Tohoku University, Sendai 980-8577, Japan
}

\begin{abstract}
We present a theoretical study of heat transport in electrically insulating ferromagnetic wires containing a domain wall. In the regime of validity of continuum micromagnetism a domain wall is found to have no effect on the heat conductance. However, spin waves are found to be reflected by domain walls with widths of a few lattice spacings, which is associated with emergence of an additional spin wave bound state. The resulting domain wall heat conductance should be significant for thin films of Yttrium Iron Garnet with sharply defined magnetic domains.
\end{abstract}

PACS numbers: 75.30.Ds, 75.60.Ch, 85.75.-d

Spin wave (SW) excitations in magnetic systems have been studied for many decades, but due to recent progress in the fabrication of magnetic nanostructures, novel detection techniques, and new discoveries such as currentinduced magnetization dynamics, the field is very much alive [1]. It has been shown that SW can deliver signal information and probe magnetic properties. Several device concepts have been proposed to use SW logic devices [2, 3]. Recent experiments show that three crucial material parameters, namely, the spin polarization $P$, the intrinsic Gilbert damping $\alpha$, and the dissipative correction to adiabaticity $\beta$, can be simultaneously determined by measuring the current-induced SW Doppler effect [4, 5]. Non-volatile data storage devices [6] and logic circuits 7] make use of magnetic domain walls (DWs). The combination of both strategies is promising as well [8]. Several phenomenon based on the interaction between SWs and DWs have been proposed within the framework of continuum micromagnetic theory, such absence of reflection [9] but an associated scattering phases shift [8, 9] of SWs by a DW, frequency doubling [10], domain wall drift [1] 13], and magnonic spin transfer torque [14 16]. A complication that has received much less attention is the breakdown of the continuum approximation of magnetization in materials with high anisotropy, in which the DW width $\delta$ can be as small as a few lattice constants $a$ 17]. Atomic-scale DWs can display very different static and dynamic properties than predicted by a continuum model [18]. For example, discrete DW jumps that match the lattice periodicity have been observed by Novoselov et al. in thin films of yttrium-iron garnet (YIG) that support DWs as narrow as $\delta / a \approx 6 / \pi[19]$. Only one attempt to formulate the theory of SWs propagating through ultranarrow DWs is known to us [20], predicting that a ferromagnetic SW can pass through a DW with little reflection if its wavelength is less than twice the thickness of the wall [20], which does not agree with our findings reported here.

In this Letter, we address DWs in wires of insulating ferromagnets such a YIG that have superior magnetic quality factors, with damping constants that can be three

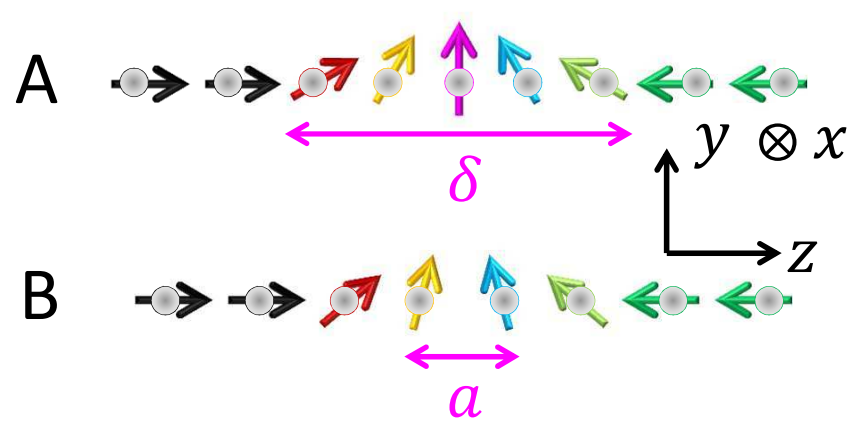

FIG. 1: (Color online) Principle ground state spin configurations for a symmetric DW: its center either coincides with one of the lattice sites (type A) or lies between them (type B). $\delta$ is the domain wall width and $a$ the lattice constant.

orders of magnitude smaller than those in metallic magnets. Their application to novel memory concepts therefore appears attractive. Charge current-induced DW motion is ruled out, of course, but heat currents can serve identical purposes by virtue of the thermal spin-transfer torque [14, 21]. Here we study SW excitations in ferromagnetically coupled spin chains in the presence of a DW, predicting remarkable effects of the discrete lattice on the SW transmission. While we confirm previous results that in a continuum model for DWs the SW transmission probability is unity, spin wave reflection in a discrete spin model is found to be finite for abrupt domain walls. The resulting domain wall heat conductance should help to detect and manipulate DWs in insulating ferromagnetic wires.

We consider a wire that is sufficiently thin such that the lateral degrees of freedom are frozen out and a 1D model is appropriate. Our model is a classical Heisenberg spin chain with local anisotropy and nearest-neighbor ferromagnetic exchange interaction, which is appropriate for materials such as YIG which has a large effective spin $(S \approx 14.3)$ per unit cell 22 ]. In the Hamiltonian 23]

$$
\mathcal{H}=-J \sum_{\langle n, m\rangle} \vec{S}_{n} \cdot \vec{S}_{m}-D \sum_{n}\left(S_{n}^{z}\right)^{2},
$$


the first term describes the nearest neighbor interaction with ferromagnetic coupling $J>0$, while the second terms is a local easy-axis anisotropy in the $z$-direction with $D>0$. The local spin variable $\vec{S}_{n}$ is a threecomponent unit vector on the $n$-th lattice site. A transverse hard axis (shape) anisotropy causes complicated spin wave dispersions in thin films. In our onedimensional system, the dipolar interaction can be important as well, but only leads to an increased easy-axis anisotropy [23, 24]. For materials with a large unit cell like YIG, our approximation to integrate the magnetic moment distribution in a single unit cell and replace them with one classical spin is a good approximation at not too high temperatures [25]. In insulating ferromagnets, the heat current is associated with both phonon and magnon channels. In YIG the magnons, responsible for interactions with DW of interest here, contribute $2 / 3$ of the total heat current at low temperatures [26, 27]. We consider a magnetic wire of length $L$ that connects two large heat reservoirs held at two temperatures $T_{L}, T_{R}$, with constant difference $\Delta T=\left(T_{L}-T_{R}\right)>0$. The magnon-mediated heat current carried by the spin chain is given by the Landauer-Büttiker formula 28]

$$
\begin{aligned}
\dot{Q} & =\frac{1}{L} \int d k \rho_{k} v_{k} \hbar \omega_{k}\left|t_{k}\right|^{2}\left[n_{B}\left(\omega_{k}, T_{L}\right)-n_{B}\left(\omega_{k}, T_{R}\right)\right] \\
& =\frac{1}{2 \pi} \int d \omega \hbar \omega\left|t_{k(\omega)}\right|^{2}\left[n_{B}\left(\omega, T_{L}\right)-n_{B}\left(\omega, T_{R}\right)\right]
\end{aligned}
$$

Here $\rho_{k}=L / 2 \pi$ is the magnon density of states, $v_{k}=\partial \omega / \partial k$ is the SW group velocity, $\hbar \omega_{k}=$ $2 D+2 J[1-\cos (k a)]$ is the SW spectrum, $t_{k}$ is the SW transmission coefficient, and $n_{B}\left(\omega, T_{L, R}\right)=$ $1 /\left(e^{\hbar \omega /\left(k_{B} T_{L, R}\right)}-1\right)$ is the Bose-Einstein distribution. In the absence of DWs, $t_{k}=1$ and for a small temperature bias

$$
\dot{Q}_{1} \doteq \frac{\Delta T}{2 \pi} \int d \omega \hbar \omega \frac{\partial n_{B}(\omega, T)}{\partial T},
$$

where $T=\left(T_{L}+T_{R}\right) / 2$.

The heat current in the presence of DW is determined by the wall profile which is obtained by energy minimization with respect to the polar angle $\theta_{n}$ of $\vec{S}_{n}$ with the easy axis:

$$
\sin \left(\theta_{n}-\theta_{n-1}\right)-\sin \left(\theta_{n+1}-\theta_{n}\right)+\frac{D}{J} \sin \left(2 \theta_{n}\right)=0
$$

where we chose that boundary conditions that spins are oriented in the $\pm z$ directions at the ends of the wire. The ground state DW configuration can be computed iteratively [29]. To second order in the small parameter $D / J \ll 1$ this procedure yields the Walker solution $\ln \tan (\theta / 2)=z / \delta$ with DW width $\delta=a \sqrt{J /(2 D)}[30$. In a typical Walker DW, labeled A (metastable) in Fig. 1 , the spin of the central lattice site is oriented normal to the easy axis. However, numerical calculation leads to a stable type B wall (Fig. 1) which has always lower energies [18]. Clearly, this difference is immaterial in the continuum limit for the magnetization (micromagnetics), and it is only relevant for atomically sharp domain walls. We will discuss below that the atomic-scale magnetization texture is observable in the heat and spin transport properties.

Proceeding from the ground state DW configuration we can construct, linearize, and solve the equations of motion $d \vec{S}_{n} / d t=-\vec{S}_{n} \times\left(-\delta \mathcal{H} / \delta \vec{S}_{n}\right)$ for the spins at a site $n$ in order to determine the frequencies and amplitudes of the allowed SW modes. This can be done conveniently in a coordinate system rotated about the $x$ axis such that the transformed spins at equilibrium point to the new $Z$-axis [31], where the spin vectors in the two coordinate systems are related as

$$
\left(\begin{array}{c}
S_{n}^{x} \\
S_{n}^{y} \\
S_{n}^{z}
\end{array}\right)=\left(\begin{array}{ccc}
1 & 0 & 0 \\
0 & \cos \theta_{n} & \sin \theta_{n} \\
0 & -\sin \theta_{n} & \cos \theta_{n}
\end{array}\right)\left(\begin{array}{c}
S_{n}^{X} \\
S_{n}^{Y} \\
S_{n}^{Z}
\end{array}\right)
$$

The low-energy excitations correspond now to a smallangle precession around the $Z$-axis with $S_{n}^{Z} \sim 1$ [16, 32]. The equations of motion for the SW amplitude at site $n$ and frequency $\omega$ in the rotated frame read

$$
\begin{aligned}
-i \frac{\omega}{J} S_{n}^{X} & =t_{n-1} S_{n-1}^{Y}+t_{n} S_{n+1}^{Y} \\
& -\left[t_{n-1}+t_{n}-\frac{2 D}{J}\left(\sin ^{2} \theta_{n}-\cos ^{2} \theta_{n}\right)\right] S_{n}^{Y} \\
-i \frac{\omega}{J} S_{n}^{Y} & =-S_{n-1}^{X}-S_{n+1}^{X} \\
& +\left(t_{n-1}+t_{n}+\frac{2 D}{J} \cos ^{2} \theta_{n}\right) S_{n}^{X}
\end{aligned}
$$

where $t_{n}=\cos \left(\theta_{n}-\theta_{n+1}\right)$. We obtain the eigenfrequencies $\omega$ and amplitudes $S_{n}^{X}, S_{n}^{Y}$ numerically.

$D / J=0.001$ leads to a broad DW profile (solid line in Fig. 2(a)) that cannot be distinguished from the Walker solution. The SW mode profile $S_{n}^{Y}$ is also plotted (circles), with the corresponding frequency given in the plot. For a broad DW the SW profile agrees very well with the analytical solution (dashed line in Fig. 2(a)) of the continuum model [14] governed by the Schrödinger equation

$$
\left[-\frac{d^{2}}{d \xi^{2}}+V(\xi)\right] \varphi(\xi)=q^{2} \varphi(\xi)
$$

with $\xi=z / \delta, \varphi=S_{X}-i S_{Y}, q=k \delta$ with spin-wave vector $k$, and potential $V(\xi)=-2 \operatorname{sech}^{2} \xi$. The potential obtained here originates from the non-commutativity of the gauge transformation with the momentum operator [16], similar to electron transport in magnetic textures 33]. However, in contrast to electrons, SWs do not feel a vector potential and only a scalar potential remains. The solutions of Eq. (77) are one bound SW 


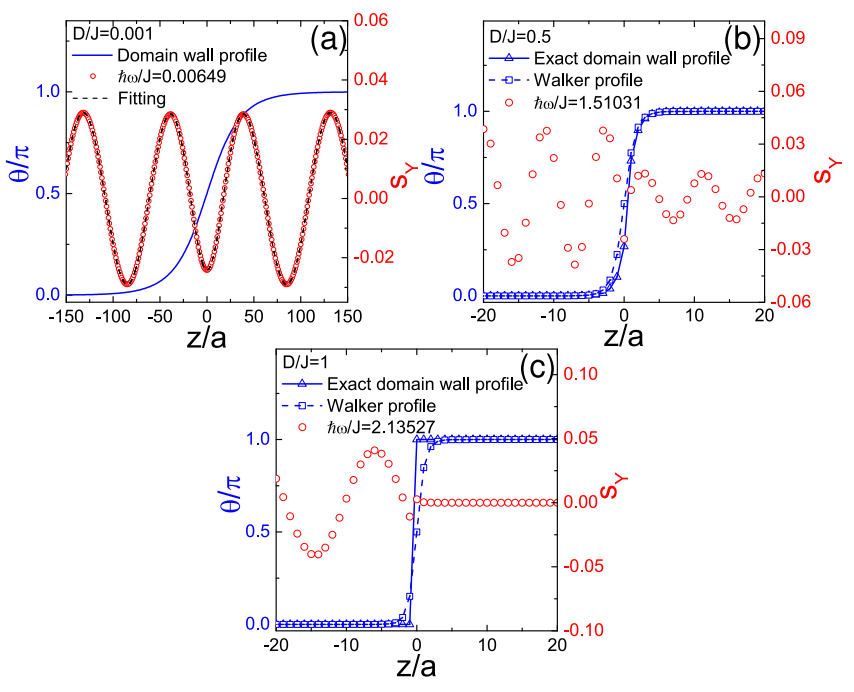

FIG. 2: (Color online) (a) The magnetization profile of a wide head-to-head DW (solid line). The numerical SW solution (circles) for frequency $\omega$ in a linear ferromagnetic chain cannot be distinguished from the solution (dashed line) for the continuum model. (b) A computed narrow DW profile (triangles) compared with the Walker model (squares). The SW amplitude for a selected frequency is shown by circles. (c) The ground state profile of an abrupt DW (triangles) compared with the Walker profile (squares). The amplitude of a SW for another given frequency is shown by circles.

state and propagating SWs that are not reflected at the DW but pick up a wave vector $k$-dependent phase shift $\eta(k)=2 \tan ^{-1}\{1 /(k \delta)\}$ [14 that modifies the frequency dispersion [34] and causes interference effects in rings [8]. Since the DW transmission coefficient is $t_{k}=e^{i \eta(k)}$ and the transmission probability $\left|t_{k}\right|^{2}=1$, there is no effect on DC heat transport. This conclusion is not modified by a transverse hard-axis anisotropy $D_{\perp} \sum_{n}\left(S_{n}^{x}\right)^{2}$, since propagating SWs are elliptical but still reflectionless. The conclusion holds in spite of the bound magnon state at the DW and in contrast to conducting ferromagnets, where the DW leads to an increase of electric resistivity even in the continuum model [35]. In order to detect such a DW bound state in insulating ferromagnets, one has to resort to spectroscopic or other techniques [36].

The continuum approach breaks down for atomic scale DWs such as in Fig. 1. The profiles of a relatively narrow DW for both exact numerical (triangles) and Walker solution (squares) are shown in Fig. 2(b) for a large anisotropy $D / J=0.5$. We see that now spin waves do get reflected! Figure 2(c) shows the configuration of an abrupt DW (triangles), with $D / J=1$. The Walker profile is also plotted for comparison (squares). Interestingly, we now find that all SWs (circles) are totally reflected. The physics is explained below.

As pointed out before, the DW supports bound SW states. Figure 3(a) shows the numerical results for the
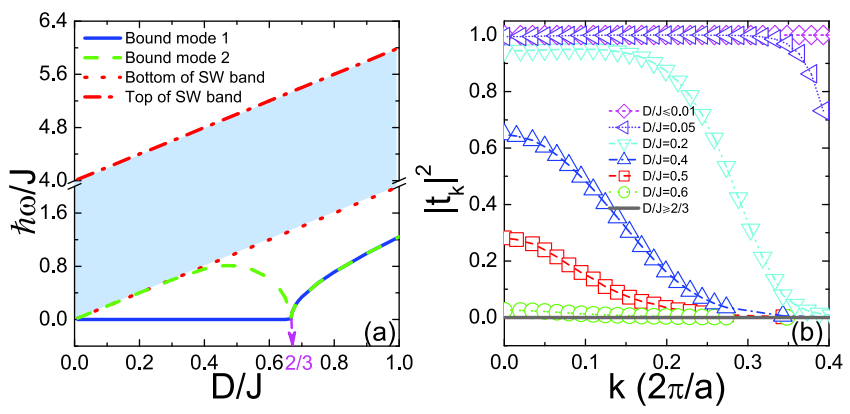

FIG. 3: (Color online) (a) Frequencies of DW bound states and SW continuum (shaded area between dotted and dashdotted lines). The solid curve represents the lowest mode, while the dashed curve shows the second bound state. (b) $k$-dependence of SW transmission for different values of $D / J$.

bound state frequencies as a function of $D / J$ below the continuum SW region, where the dotted line delineates the SW gap $\left(\hbar \omega_{g}=2 D\right)$ and the dash-dotted line the top of the SW band $\left(\hbar \omega_{t}=2 D+4 J\right)$. We confirm the existence of a transition point at $D / J=2 / 3$ that separates smooth and sharp DW configurations [37] but without assuming $\left|\theta_{n \pm 1}-\theta_{n}\right| \ll 1$, which is clearly not the case for these narrow DWs. The transition point obtained here differs from the value $D / J=5 / 9$ calculated in Ref. [38]. Bound state 1 (solid curve), below the transition point $\omega_{b 1}=0$ is caused by the rotational symmetry of the smooth DW about the $z$ axis. When $D / J>2 / 3$ the DW width is the lattice constant and bound state 1 becomes degenerate with bound state 2 (dashed curve), i.e., $\omega_{b 1}=\omega_{b 2}$, with frequency monotonously increasing with $D / J$. For wide DWs, i.e., $D / J \ll 2 / 3$, bound state 2 merges with the continuum states. As the DW becomes narrower (increasing $D / J$ ), $\omega_{b 2}$ splits from the SW continuum and displays a non-monotonic dependence on $D / J$, with maximum at $\hbar \omega_{b 2} \approx 0.47 D$, dropping to zero when $D / J \rightarrow 2 / 3$.

We can now understand why the SW is reflected at ultra-narrow DWs. Let us consider a confining potential $V(\xi) \rightarrow 0$ as $|\xi| \rightarrow \infty$ for which the eigenvalue problem Eq. (77) has $N$ bound states with $q=i \kappa_{n}, n=1,2, \ldots, N$, where $\kappa_{n}$ is real. Through the inverse scattering formalism, one can construct a general $2 N$-parameter formula for a reflectionless potential with $N$ bound states [39],

$$
V(\xi)=-2 \frac{d^{2}}{d \xi^{2}} \ln \operatorname{det} A(\xi),
$$

where the matrix $A_{m n}(\xi)=\delta_{m n}+\lambda_{m} \lambda_{n} /\left(\kappa_{m}+\kappa_{n}\right)$, with Kronecker function $\delta_{m n}$ and $\lambda_{n}(\xi)=c_{n} e^{-\kappa_{n} \xi}$ is symmetric. For $N=1$ we recover the previous result of the continuum model [14]. In the discrete model, however, $N=2$. Figure 3(a) clearly shows that the bound state 2 vanishes into the SW continuum only for wide DWs $(D / J \ll 2 / 3)$. Only in this limit there is just 
one bound state (mode 1 ) and the DW profile is perfectly described by the reflectionless Walker model. To model the profile of a narrow DW, we expand Eq. (4) to the fourth order, and obtain the DW configuration $\ln \tan (\theta / 2)=(z / \delta)+(1 / 24)(a / \delta)^{2}[6 \tanh (z / \delta)-z / \delta]$ leading to the confining potential $\tilde{V}(\xi)=-2 \operatorname{sech}^{2}[y(\xi)]$ with $y(\xi)=\xi+(1 / 24)(a / \delta)^{2}(6 \tanh \xi-\xi)$. According to Thacker et al. 39], a reflectionless potential with two bound states must obey Eq. (8). However, the confining potential induced by a narrow DW as derived above cannot be described by a four-parameter reflectionless potential Eq. (8), and indeed necessarily induces reflections. An expansion up to higher order will not change this conclusion. Therefore, the SW must lose its property of total transmission, as confirmed by our numerical results.

The $k$-dependent SW transmission for different parameters $D / J$ are shown in Fig. 3(b), where the $k$ values are obtained by fast Fourier transformation of the computed SW mode amplitudes [24]. The wave vectors $k$ of interest range here from zero up to $0.8 \pi / a$ corresponding to a very short wavelength $(2.5 a)$ beyond which a classical spin model might become unreliable [40]. In agreement with previous results there is no detectable reflection in the calculations with $D / J<0.01$. However, for a large anisotropy, the SW will be only partially transmitted through the DW, with transmission probability monotonically decreasing with increasing wave vector $k$. These results are very different from those obtained with a local spin-spiral approximation, i.e., a constant pitch within the DW width [41], which leads to perfect transmission for SWs with large $k$, but hindered propagation at long wave lengths [20], which is caused by the unphysical kinks in the magnetization texture of that model. We also have a message for micromagnetic simulations in which the continuum Hamiltonian $\mathcal{H}=J^{\prime}(\partial \mathbf{m} / \partial z)^{2} / 2-D m_{z}^{2}$ is discretized into block spins $J^{\prime} \partial^{2} \mathbf{m}_{n} / \partial z^{2}=J^{\prime}\left(\mathbf{m}_{n-1}-2 \mathbf{m}_{n}+\mathbf{m}_{n+1}\right) /(\Delta z)^{2}$. The mesh size $\Delta z$ is found here to be rather critical, since SW reflections that are artifacts in a continuum model occur unless $\pi \sqrt{J^{\prime} / 2 D} \geqslant 22 \Delta z$.

Our results should be relevant for systems with close to atomically sharp DW such as YIG thin films. In the case of a narrow atomic-scale DW, the spin wave gets reflections which leads to a modification to the heat transport as $\dot{Q}_{2}=\dot{Q}_{1}+\Delta \dot{Q}$, where

$$
\Delta \dot{Q}=\frac{\Delta T}{2 \pi} \int d \omega\left(\left|t_{\omega}\right|^{2}-1\right) \hbar \omega \frac{\partial n_{B}(\omega, T)}{\partial T} .
$$

We can see that the DW decreases the heat conductance since $\Delta \dot{Q}<0$. We can estimate the reduction of the heat current due to magnon reflection at low temperatures as follows: magnons then exist only the bottom of SW band $(k=0)$ at which $|t(k=0)|^{2}$ can be used to estimate the total transmission probability and $\Delta \dot{Q} / \dot{Q}_{1} \approx|t(k=0)|^{2}-1$. When $D / J=0.4$,
$\Delta \dot{Q} / \dot{Q}_{1} \sim-35 \%$. At room temperature $k_{B} T$ is larger than the exchange energy $J \approx 40 \mathrm{~K}$ in YIG [22, 25] and magnons with high wave numbers become important for the heat transport. The spin wave reflection becomes significant when $\delta \lesssim(10 / \pi) a$, where $a=1.24 \mathrm{~nm}$ in YIG $[19,22,25]$.

Ultranarrow DWs are sensitive to crystal lattice pinning: the $\mathrm{A}$ and B-type walls in the 1D model (Fig. 1) have different ground state energies [18]. A domain wall therefore has to surmount an energy barrier when moving from one atomic plane to another in a "Peierls" potential and a threshold external force is necessary to assist the DW motion [18]. Novoselov et al. [19] indeed observed a critical magnetic field for field-driven atomic-scale DW dynamics. Consequently, an intrinsic critical heat/spin current exists for the propagation of DWs even in perfect wires. According to Ref. [15], a temperature gradient of $1 \mathrm{~K} / \mathrm{nm}$ creates a pressure corresponding to a field $H=5 \mathrm{mT}$ for a magnetic insulator with a saturation magnetization $M_{s}=2 \times 10^{6} \mathrm{~A} / \mathrm{m}$. We estimate the critical temperature gradient to overcome the atomic pinning as $0.1 \mathrm{~K} / \mu \mathrm{m}$ for the critical magnetic field value $H_{c}=0.7 \mathrm{G}$ and $M_{s}=1.6 \times 10^{4} \mathrm{~A} / \mathrm{m}$ reported in Ref. [19].

In conclusion, we studied the heat transport by SWs through magnetic DWs. When the DW becomes the order of a few lattice constants a second bound state emerges that is absent in the continuum approximation and causes strong SW reflection. The SW reflection leads to a DW heat conductance. The results developed here are relevant for ultrahigh-density storage devices based on DWs [42] when based on insulating ferromagnets.

We thank Akashdeep Kamra for helpful discussion. This work is supported by the FOM foundation, DFG Priority Program 1538 SpinCat, and EG-STREP MACALO.

[1] V.V. Kruglyak, S.O. Demokritov, and D. Grundler, J. Phys. D: Appl. Phys. 43, 260301 (2010); B. Lenk, H. Ulrichs, F. Garbs, and M. Münzenberg, Phys. Rep. 507, 107 (2011).

[2] T. Schneider, A.A. Serga, B. Leven, B. Hillebrands, R.L. Stamps, and M.P. Kostylev, Appl. Phys. Lett. 92, 022505 (2008).

[3] A. Khitun, M.Q. Bao, and K.L. Wang, IEEE Trans. Magn. 44, 2141 (2008).

[4] V. Vlaminck and M. Bailleul, Science 322, 410 (2008).

[5] K. Sekiguchi, K. Yamada, S.M. Seo, K.J. Lee, D. Chiba, K. Kobayashi, and T. Ono, Phys. Rev. Lett. 108, 017203 (2012).

[6] S.S.P. Parkin, M. Hayashi, and L. Thomas, Science 320, 190 (2008).

[7] D.A. Allwood, G. Xiong, C.C. Faulkner, D. Atkinson, D. Petit, and R.P. Cowburn, Science 309, 1688 (2005).

[8] R. Hertel, W. Wulfhekel, and J. Kirschner, Phys. Rev. 
Lett. 93, 257202 (2004).

[9] C. Bayer, H. Schultheiss, B. Hillebrands, and R.L. Stamps, IEEE Trans. Magn. 41, 3094 (2005).

[10] S.J. Hermsdoerfer, H. Schultheiss, C. Rausch, S. Schäfer, B. Leven, S.K. Kim, and B. Hillebrands, Appl. Phys. Lett. 94, 223510 (2009).

[11] A.V. Mikhaı̌lov and A.I. Yaremchuk, JETP Lett. 39, 354 (1984).

[12] D.S. Han, S.K. Kim, J.Y. Lee, S.J. Hermsdoerfer, H. Schultheiss, B. Leven, and B. Hillebrands, Appl. Phys. Lett. 94, 112502 (2009).

[13] M. Jamali, H. Yang, and K.J. Lee, Appl. Phys. Lett. 96, 242501 (2010).

[14] P. Yan, X.S. Wang, and X.R. Wang, Phys. Rev. Lett. 107, 177207 (2011).

[15] D. Hinzke and U. Nowak, Phys. Rev. Lett. 107, 027205 (2011).

[16] A.A. Kovalev and Y. Tserkovnyak, Europhys. Lett. 97, 67002 (2012).

[17] C.A. Jenkins and D.I. Paul, J. Appl. Phys. 111, 013915 (2012).

[18] H.R. Hilzinger and H. Kronmüller, Phys. Status Solidi 54, 593 (1972).

[19] K.S. Novoselov, A.K. Geim, S.V. Dubonos, E.W. Hill, and I.V. Grigorieva, Nature (London) 426, 812 (2003).

[20] S.H. Liu, J. Magn. Magn. Mater. 12, 262 (1979).

[21] M. Hatami, G.E.W. Bauer, Q. Zhang, and P.J. Kelly, Phys. Rev. Lett. 99, 066603 (2007).

[22] I.S. Tupitsyn, P.C.E. Stamps, and A.L. Burin, Phys. Rev. Lett. 100, 257202 (2008); A. Kreisel, F. Sauli, L. Bartosch, and P. Kopietz, Eur. Phys. J. B 71, 59 (2009).

[23] R. Wieser, U. Nowak, and K.D. Usadel, Phys. Rev. B 69, 064401 (2004).

[24] R. Wieser, E.Y. Vedmedenko, and R. Wiesendanger, Phys. Rev. B 81, 024405 (2010).

[25] V. Cherepanov, I. Kolokolov, and V. L'vov, Phys. Rep.
229, 81 (1993).

[26] J.E. Rives, G.S. Dixon, and D. Walton, J. Appl. Phys. 40, 1555 (1969).

[27] R.L. Douglass, Phys. Rev. 129, 1132 (1963).

[28] F. Meier and D. Loss, Phys. Rev. Lett. 90, 167204 (2003).

[29] E.E. Fullerton, J.S. Jiang, M. Grimsditch, C.H. Sowers, and S.D. Bader, Phys. Rev. B 58, 12193 (1998).

[30] N.L. Schryer and L.R. Walker, J. Appl. Phys. 45, 5406 (1974).

[31] G. Tatara and H. Fukuyama, Phys. Rev. Lett. 72, 772 (1994).

[32] V.K. Dugaev, P. Bruno, B. Canals, and C. Lacroix, Phys. Rev. B 72, 024456 (2005); K.Y. Guslienko, G.R. Aranda, and J.M. Gonzalez, Phys. Rev. B 81, 014414 (2010).

[33] A. Brataas, G. Tatara, and G.E.W. Bauer, Phys. Rev. B 60, 3406 (1999).

[34] R. Wieser, E.Y. Vedmedenko, and R. Wiesendanger, Phys. Rev. B 79, 144412 (2009).

[35] A.V. Ferrer, P.F. Farinas, and A.O. Caldeira, Phys. Rev. Lett. 91, 226803 (2003).

[36] T. Koma and M. Yamanaka, Phys. Rev. B 65, 104434 (2002).

[37] B. Barbara, J. Magn. Magn. Mater. 129, 79 (1994).

[38] A. Vindigni, Inorg. Chim. Acta 361, 3731 (2008).

[39] H.B. Thacker, C. Quigg, and J.L. Rosner, Phys. Rev. D 18, 274 (1978).

[40] J. Prokop, W.X. Tang, Y. Zhang, I. Tudosa, T.R.F. Peixoto, Kh. Zakeri, and J. Kirschner, Phys. Rev. Lett. 102, 177206 (2009).

[41] R.P. van Gorkom, A. Brataas, and G.E.W. Bauer, Phys. Rev. Lett. 83, 4401 (1999).

[42] R. Nebashi, N. Sakimura, Y. Tsuji, S. Fukami, H. Honjo, S. Saito, S. Miura, N. Ishiwata, K. Kinoshita, T. Hanyu, T. Endoh, N. Kasai, H. Ohno, and T. Sugibayashi, IEEE Symp. VLSI Circuit Dig. Tech. Pap. 300 (2011). 